\title{
Maximizing the Lifetime of Wireless Sensor Networks with Mobile Sink in Delay-Tolerant Applications
}

\author{
YoungSang Yun and Ye Xia \\ Computer and Information Science and Engineering Department, \\ University of Florida, Gainesville, FL 30611-6120 \\ email: $\{$ yyun, yx1\}@cise.ufl.edu
}

\begin{abstract}
This paper proposes a framework to maximize the lifetime the wireless sensor network (WSN) by using a mobile sink when the underlying applications tolerate delayed information delivery to the sink. Within a prescribed delay tolerance level, each node does not need to send the data immediately as they become available. Instead, the node can store the data temporarily and transmit them when the mobile sink is at the most favorable location for achieving the longest WSN lifetime. To find the best solution within the proposed framework, we formulate optimization problems that maximize the lifetime of the WSN subject to the delay bound constraints, node energy constraints and flow conservation constraints. We conduct extensive computational experiments on the optimization problems and find that the lifetime can be increased significantly as compared to not only the stationary sink model but also more traditional mobile sink models. We also show that the delay tolerance level does not affect the maximum lifetime of the WSN.
\end{abstract}

\section{INTRODUCTION}

A wireless sensor network (WSN) consists of sensor nodes capable of collecting information from the environment and communicating with each other via wireless transceivers. The collected data will be delivered to one or more sinks, generally via multi-hop communication. The sensor nodes are typically expected to operate with batteries and are often deployed to not-easily-accessible or hostile environment, sometimes in large quantities. It can be difficult or impossible to replace the batteries of the sensor nodes. On the other hand, the sink is typically rich in energy. Since the sensor energy is the most precious resource in the WSN, efficient utilization of the energy to prolong the network lifetime has been the focus of much of the research on the WSN.

Although the lifetime of the WSN can be defined in many ways, we adopt the definition that it is the time until the first node exhausts its energy, which is a widely used. Much work has been done during recent years to increase the lifetime of the WSN. Among them, in spite of the difficulties in realization, taking advantage of the mobility in the WSN has attracted much interests from researchers [1], [2], [3], [4], [5], [6], [7], [8], [9]. We can take the mobile sink as an example of mobility in the WSN. The communications in the WSN has the many-to-one property in that data from a large number of sensor nodes tend to be concentrated into a few sinks. Since multi-hop routing is generally needed for distant sensor nodes from the sinks to save energy, the nodes near a sink can be burdened with relaying a large amount of traffic from other nodes. This phenomenon is sometimes called the "crowded center effect" [10] or the "energy hole problem" [11], [12], [13]. It results in energy depletion at the nodes near the sink too soon, leading to the separation of the sink from the rest of nodes that still have plenty of energy. However, by moving the sink in the sensor field, one can avoid or mitigate the energy hole problem and expect an increased network lifetime.

This paper proposes a framework to maximize the lifetime of the WSN by taking advantage of the mobile sink. Compared with other mobile-sink proposals, the main novelty is that we consider the case where the underlying applications tolerate delayed information delivery to the sink. In our proposal, within a prescribed delay tolerance level, each node does not need to send the data immediately as they become available. Instead, the node can store the data temporarily and transmit them when the mobile sink is at the stop most favorable for achieving the longest network lifetime. To find the best solution within the proposed framework, we formulate optimization problems that maximize the lifetime of the WSN subject to the delay bound constraints, node energy constraints and flow conservation constraints. Another one of our contributions is that we compare our proposal with several other lifetime-maximization proposals and quantify the performance differences among them. Our computational experiments have shown that our proposal increases the lifetime significantly when compared to not only the stationary sink model but also more traditional mobile sink models. We also show that the delay tolerance level does not affect the maximum lifetime of the WSN.

Our proposal is more sophisticated than most previous lifetime-improvement proposals that we know of. It integrates the following energy-saving techniques, multipath routing, a mobile sink, delayed data delivery and active region control, into a single optimization problem. Such sophistication comes at a cost. Whether the proposal should be adopted in practice will depend on the tradeoff between the lifetime gain and the actual system cost. Even if the decision is not to adopt it due to a high cost or high complexity, the framework in the 
paper is still useful because it can supply the practitioners with a performance benchmark, e.g., how much lifetime improvement opportunity there is. By also formulating the optimization problems related to other proposals and providing cross comparison, the paper provides extra convenience for comparing and understanding different proposals.

We now briefly review the most relevant work on how to exploit mobility to increase the network lifetime. In [4], the authors introduced the mobile agents called MULEs, which move around and collect data from nearby sensor nodes on behalf of the immobile sink. When the mobile agents move to the vicinity of the sink, they forward the collected data to the sink. The mobile agents are assumed to have plenty of energy. The movement of each mobile agent is modeled as a random walk. It was shown that the queues in the mobile agents and the sensor nodes are finite and the delay of the collected data is bounded. However, the authors did not show the quantitative improvement of the network lifetime by using mobile agents.

In [1], the authors formulated a linear programming problem to maximize the lifetime of a WSN where the sensor nodes are deployed in a grid pattern and the sink can move to a subset of the grid points. When the location of the mobile sink is known to the sensor nodes, each node can identify the minimum hop-count paths to the sink. The nodes distribute the data evenly onto these paths.

The authors of [3] showed that the network lifetime can be extended significantly if the mobile sink moves around the periphery of the WSN. They assumed that, if the mobile sink can balance the traffic load of the nodes, the lifetime of the network can increase. Therefore, they proposed an optimization problem for choosing a mobility strategy that minimizes the maximum traffic load of the nodes. However, they assumed the shortest path routing, which, in general, does not produce the best lifetime.

The problem of finding the sink movement path to optimize the lifetime of the WSN, as in [5], is hard to solve. This type of problems generally belong to the traveling salesman problem. In [9], the authors studied how to find the optimal sink stops and the schedule of visit to the each of the stops. If the candidate locations for the stops are unconstrained, this problem is also NP-hard. However, if the stops are constrained to be selected from a finite set of known locations, the problem can be easily formulated into linear programming. They proposed an approximation algorithms to the unconstrained problem by properly dividing the whole sensor field into a finite number of disjoint small areas, and then, converted the unconstrained problem into a constraint problem. However, to obtain a good approximation ratio, the number of small areas can potentially be very large, making the linear programming computation time-consuming. Therefore, in this paper, we restrict the set of potential sink stops to be from a small number of given locations rather than from arbitrary locations.

The WSN model proposed in [7] is close to ours. The authors studied the maximum lifetime problem of the WSN where the mobile sink can visit only small number of locations. They showed that the lifetime can be further increased by optimizing not only the schedule of sink visits but also routing of the traffic. However, they did not consider applications where delayed information delivery is allowed.

The rest of the paper is organized as follow. Section II describes various related lifetime maximization problems that we will compare against. The mathematical formulations of the models are provided for the purpose of comparison. In section III, we propose two novel models with a mobile sink and delayed information delivery. We show some nice properties that our models possess. In section IV, we compare our models with others by simulation and numerical experiments. The conclusions are given in Section $\mathrm{V}$.

\section{Related Lifetime Maximization Problems}

In this section, we discuss related lifetime maximization problems that have been published in the literature. We will later compare their performance with our new proposal.

First, we will describe the general assumptions about the WSN models. Let the set of sensor nodes be denoted by $\mathcal{N}$. For experimental convenience, we suppose they are uniformly randomly deployed into a circular area with radius $R$. Let the center of the disk be the origin. Each node $i$ is assumed to generate data at a constant rate of $d_{i}$ during its life span and the initial energy of $i$ is denoted by $E_{i}$. Furthermore, the nodes have the ability of adjusting their transmission power level to match the transmission distance. Similar to [14], the energy required per unit of time to transmit data at the rate of $x_{i j}$ from node $i$ to $j$ can be determined as follows.

$$
E_{i j}^{t}=C_{i j}^{t} \cdot x_{i j},
$$

where $C_{i j}^{t}$ is the required energy for transmitting one unit of data from node $i$ to $j$ and it can be modeled as follows [15].

$$
C_{i j}^{t}=\alpha+\beta \cdot d(i, j)^{e}
$$

where $d(i, j)$ is the Euclidean distance between node $i$ and $j, \alpha$ and $\beta$ are nonnegative constants, and $e$ is the path loss exponent. Typically, $e$ is in the range of 2 to 6 , depending on the environment. Here, the energy cost per unit of data does not depend on the link rate, and this is valid for the low rate regime. Hence, we need to assume that the traffic rate $x_{i j}$ is sufficiently small compared to the capacity of the wireless link.

The energy consumed at node $i$ per unit of time for receiving data from node $k$ is given by [14]

$$
E_{k i}^{r}=\gamma \cdot x_{k i}
$$

where $\gamma$ is a given constant. Hence the total energy consumption per unit time at node $i$ is

$$
\sum_{j \in \mathcal{N}} E_{i j}^{t}+\sum_{k \in \mathcal{N}} E_{k i}^{r}=\sum_{j \in \mathcal{N}} C_{i j}^{t} \cdot x_{i j}+\sum_{k \in \mathcal{N}} \gamma \cdot x_{k i}
$$

We assume that each sensor node has the same transmission range. We define the neighbors of node $i$ as $N(i)=\{j \in$ $\mathcal{N} \mid d(i, j) \leq \bar{d}\}$, when the transmission range is $\bar{d}$. 


\section{A. Static Sink Model}

In the static sink model (SSM), the sink is located at the origin and remains stationary during the operation of the WSN. Data originated from the sensor nodes flow into the sink in a multi-hop fashion. As soon as the data becomes available at a node, it gets transmitted toward the sink. Typically, the rate at which each sensor node $i$ harvests data from the outside world is a constant. We denote it by $d_{i}$. The problem of maximizing the lifetime in this model is formulated as follows [16], [17].

Problem : Staic Sink Model (SSM)

$$
\begin{array}{ll}
\max & T \\
\text { s. t. } & \sum_{j \in N(i)} x_{i j}-\sum_{k: i \in N(k)} x_{k i}=d_{i}, \quad i, j, k \in \mathcal{N} \\
& \left(\sum_{j \in N(i)} C_{i j}^{t} \cdot x_{i j}+\sum_{k: i \in N(k)} \gamma \cdot x_{k i}\right) \cdot T \leq E_{i}, \\
& i, j, k \in \mathcal{N} \\
& \\
& x_{i j} \geq 0, \\
& T \geq 0,
\end{array}
$$

The constraint (5) is the "flow conservation constraint", which states that, at a node $i$, the sum of all outgoing flows is equal to the sum of all incoming flows plus flows generated at node $i$ itself, or $d_{i}$. The inequality (6) is the energy constraint and it means that the total energy consumed by a node during the lifetime $(T)$ cannot exceed the initial energy of the node. With this formulation, the routing is dynamic and allows multipath communications. There is no assumption on fixedpath routing, such as the shortest path routing. The above optimization problem can be easily converted into a linear programming (LP) problem.

\section{B. Mobile Sink Model}

In the mobile sink model (MSM), we assume that the sink can move around within the sensor field and stop at certain locations to gather the data from the sensor nodes. We ignore the traveling time of the sink between locations. Let $\mathcal{L}$ be the set of possible locations where the sink can stop. The sink does not necessarily stop at (i.e., stays for a positive duration) all locations in $\mathcal{L}$ in the interest of maximizing the network lifetime [1], [9]. In this model, the order of visit to the stops has no effect on the network lifetime and can be arbitrary. The sink sojourn time at a location $l \in \mathcal{L}$ is denoted by $z_{l}$; it is the time that the sink spends at $l$ to collect data from the sensor nodes. The overall network lifetime $T=\sum_{l \in \mathcal{L}} z_{l}$. To find the optimal network lifetime, we need to consider the routing of the traffic as well as the duration of stay by the sink at each stop [9], [2], [1], [7]. Let $x_{i j}^{(l)}$ be the flow rate from node $i$ to $j$ while the sink is at stop $l$. Let $\hat{\mathcal{N}}=\mathcal{N} \cup \mathcal{L}$. The lifetime

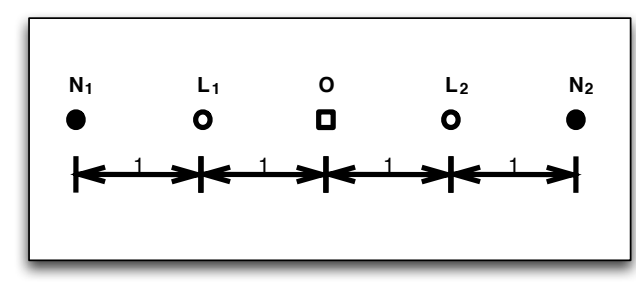

Fig. 1. Examples of the static sink model (SSM), mobile sink model (MSM), and delay tolerant mobile sink model (DT-MSM)

maximization problem can be formulated as follows.

\section{Problem : Mobile Sink Model (MSM)}

$$
\begin{aligned}
& \max T=z_{1}+z_{2}+\cdots+z_{|\mathcal{L}|} \\
& \text { s. t. } \sum_{j \in N(i)} x_{i j}^{(l)}-\sum_{k: i \in N(k)} x_{k i}^{(l)}=d_{i} \text {, } \\
& i, k \in \mathcal{N}, j \in \hat{\mathcal{N}}, l \in \mathcal{L} \\
& \sum_{l=1}^{|\mathcal{L}|} z_{l}\left(\sum_{j \in N(i)} C_{i j}^{(l)} \cdot x_{i j}^{(l)}+\sum_{k: i \in N(k)} \gamma \cdot x_{k i}^{(l)}\right) \leq E_{i}, \\
& i, k \in \mathcal{N}, j \in \hat{\mathcal{N}}, l \in \mathcal{L} \\
& x_{i j}^{(l)} \geq 0, \quad i \in \mathcal{N}, j \in \hat{\mathcal{N}}, l \in \mathcal{L} \\
& z_{l} \geq 0, \quad l \in \mathcal{L}
\end{aligned}
$$

The energy required for transmitting one unit of data when the sink is at $l$ can be expressed as,

$$
C_{i j}^{(l)}= \begin{cases}C_{i j}^{t} & \text { if } j \notin \mathcal{L} \\ \infty & \text { if } j \in \mathcal{L} \text { and } j \neq l \\ C_{i l}^{t}=\alpha+\beta \cdot d(i, l)^{e} & \text { if } j \in \mathcal{L} \text { and } j=l,\end{cases}
$$

where $C_{i j}^{t}$ is the same as in the SSM.

Constraint (9) denotes the flow conservation for all nodes when the sink is at $l$. Constraint (10) says that the total energy consumed at the node $i$ can not exceed the initial energy $E_{i}$. By multiplying (9) with $z_{l}$ and substituting $x_{i j}^{(l)} \cdot z_{l}$ with new variable $y_{i j}^{(l)}$, we have replace (9) with the following new constraint.

$$
\sum_{j \in N(i)} y_{i j}^{(l)}-\sum_{k: i \in N(k)} y_{k i}^{(l)}=z_{l} \cdot d_{i}, l \in \mathcal{L}, i \in \mathcal{N}, j \in \hat{\mathcal{N}} .
$$

Similarly, constraint (10) can be changed into

$$
\begin{array}{r}
\sum_{l=1}^{|\mathcal{L}|}\left(\sum_{j \in N(i)} C_{i j}^{t(l)} \cdot y_{i j}^{(l)}+\sum_{k: i \in N_{(k)}} \gamma \cdot y_{k i}^{(l)}\right) \leq E_{i}, \\
i \in \mathcal{N}, j \in \hat{\mathcal{N}}, l \in \mathcal{L} .
\end{array}
$$

With constraints (14), (15),(12), and non-negativity constraints of $y_{i j}^{(l)}$, the above optimization problem is converted into an LP problem. Here, $y_{i j}^{(l)}$ is interpreted as the total traffic volume for node $i$ to send to node $j$ while the sink stays at $l$. 


\section{Lifetime MaXimization IN Delay Tolerant MobILE SinK MOdel}

In this section, we consider how to maximize the network lifetime in applications that can tolerate a certain amount of delay. We call the resulting WSN model delay tolerant mobile sink model (DT-MSM). In this setting, each nodes can postpone the transmission of data until the sink is at the stop most favorable for extending the network lifetime. This way, the nodes can collectively achieve a longer network lifetime. In contrast, the SSM and MSM do not exploit this possibility.

Let $D$ be the maximum tolerable delay, or the delay tolerance level. We assume that the sink finishes one round of visit to all the stops (where the sink stays for a positive duration to collect data) in $D$ time units, and then, repeats with another round again and again. Note that two consecutive visits to the same stop takes a time $D$.

Let's take an example to show how our framework can outperform other ones. Consider the two-node example shown in Figure 1. $N_{1}$ and $N_{2}$ are two sensor nodes and $L_{1}$ and $L_{2}$ are the candidate stops of the mobile sink. Suppose we ignore the receiving energy requirement and suppose the transmission energy per unit of data is equal to the square of the distance between the sender and the receiver. Both nodes $N_{1}$ and $N_{2}$ generate data at $1 \mathrm{bps}$ and have 100 units of energy initially. If the sink is located at $O$ in the SSM, both nodes spend 4 units of energy for sending a bit of data. It is obvious that the optimal lifetime is 25 seconds. In the MSM with sink locations $\left\{L_{1}, L_{2}\right\}$, due to the symmetry of the structure, the sink stays at both $L_{1}$ and $L_{2}$ for the same amount of time to achieve the maximum lifetime. Each node spends 1 or 9 units of energy for sending 1 bit of data depending whether the sink is at $L_{1}$ or $L_{2}$. The average energy consumption per bit is 5 units. Thus, the lifetime is 20 seconds. In the DT-MSM, we assume that the sink alternates between the two stops and stays for 1 second at each stop in each cycle. Hence, $D=2$ seconds. When the sink stays at $L_{1}$, only $N_{1}$ sends 2 bits of data to the sink; when the sink moves to $L_{2}$, only $N_{2}$ transmits 2 bits of data. Both nodes spend 1 unit of energy per second on average. Thus, the lifetime is 100 seconds, a significant increase compared to the SSM and MSM. This is because, in the DT-MSM, the nodes do not always participate in the communication for all the sink stops; they each wait until the sink's location is most favorable for energy saving, and then send data at the higher rate. Recall that we have assumed that the traffic rate is sufficiently small compared to the capacity of the wireless link, and hence, sending data a higher rate does not alter the per-bit energy consumption.

Unlike the MSM or SSM, the sink in the DT-MSM can collect data from only a subset of the set of all sensor nodes, $\mathcal{N}$, at each stop. Let $R_{l}$ be the subset of $\mathcal{N}$ such that only nodes in $R_{l}$ can participate in the communication when the sink is at $l \in \mathcal{L}$. We call $R_{l}$ the coverage of the sink location $l$. Note that the union of $R_{l}$ over $l \in \mathcal{L}$ must be the set of all sensor nodes, $\mathcal{N}$. In other words, any sensor node should be covered by at least one sink location. When the node $i$ is

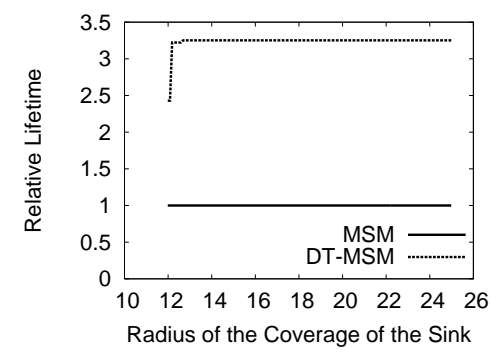

Fig. 2. Comparison of lifetimes of MSM and DT-MSM under the various radii of coverage

in $R_{l}$, node $i$ is said to be active at $l \in \mathcal{L}$. Although we can construct $R_{l}$ in many ways depending on the application of interest, in this paper, a very simple method of constructing $R_{l}$ is considered. Fix a positive number $r$. We call $r$ the radius of coverage of the sink. For each $l \in \mathcal{L}$, if $d(i, l) \leq r$, where $i \in \mathcal{N}$, then $i \in R_{l}$. Here, the radius of coverage of the sink $(r)$ should be large enough so that every sensor node belongs to at least one $R_{l}$.

In both SSM and MSM, the sink collects data from each node $i$ at the same rate at which node $i$ generates the data. However, in the DT-MSM, the data transmission rate at node $i$ during the collection time is no longer the same as the constant data generation rate $d_{i}$. When node $i$ is not active (i.e., not covered by the current sink location), it continues to gather data and should store the newly generated data. Hence, data buffering is required by our framework. Within a cycle of $D$ time units, the total stored data at each node $i$ is at most $D \cdot d_{i}$. For ease of presentation, we assume the sink visits all locations in $\mathcal{L}$ in the order of $1 \rightarrow 2 \rightarrow \cdots|\mathcal{L}| \rightarrow 1 \cdots$. The sink may stay at some location for zero time. With slight abuse of terminology, we re-define the network lifetime $T$ to be the number of cycles made by the sink until the first node dies due to energy exhaustion. The actual lifetime is $T \cdot D$. We next describe two variants of the model: the sub-flow-based model and the queue-based model.

\section{A. Sub-Flow-Based Model}

In the sub-flow-based model, the nodes in the current coverage $R_{l}$ are not allowed to buffer the relayed traffic from other nodes; as soon as a node in $R_{l}$ receives the data from other nodes, it immediately forwards the data to its neighboring nodes. To model this constraint at each node $i$, we need to differentiate the data generated by node $i$ itself and the data originally generated by other nodes but forwarded to node $i$. The data generated by the same source is sometimes called a commodity or a sub-flow [18], [19]. Let $x_{i j}^{(m, l)}$ be the rate assignment from node $i$ to the node $j$, while the sink is at $l$, for the traffic generated by node $m$ (commodity $m$ ). Let $x_{i j}^{(l)}$ be the aggregated rate of traffic that needs to be forwarded to node $j$ from node $i$ when the sink is at $l$. That is,

$$
x_{i j}^{(l)}=\sum_{m \in R_{l}} x_{i j}^{(m, l)}, \quad i \in R_{l}, j \in R_{l} \cup\{l\}, l \in \mathcal{L} .
$$


Since at node $i$, the commodity or sub-flow of other nodes $m \in R_{l}, m \neq i$ must be forwarded as soon as it has been received, we must have

$$
\begin{aligned}
\sum_{k: i \in N_{l}(k)} x_{k i}^{(m, l)} & =\sum_{j \in N_{l}(i)} x_{i j}^{(m, l)}, \\
& i, m \neq i, k \in R_{l}, j \in R_{l} \cup\{l\},
\end{aligned}
$$

where $N_{l}(i)=R_{l} \cap N(i)$. The flow conservation at node $i$ can be expressed as follows, which is the same as in the MSM except that the net amounts of traffic originated from node $i$ itself, $\left(w_{i}^{(l)}, l \in \mathcal{L}, i \in \mathcal{N}\right)$, are now decision variables.

$$
z_{l}\left(\sum_{j \in N_{l}(i)} x_{i j}^{(l)}-\sum_{k: i \in N_{l}(k)} x_{k i}^{(l)}\right)=w_{i}^{(l)} .
$$

The data buffered during the previous sink-movement cycle must be cleared in the current cycle. This requirement can be written as

$$
\sum_{l: i \in R_{l}} w_{i}^{(l)}=D \cdot d_{i}
$$

The following is the lifetime maximization problem in subflow-based DT-MSM.

\section{Problem : Sub-Flow-Based DT-MSM}

$\max T$

$$
\begin{aligned}
& \text { s. t. } \sum_{m \in R_{l}} x_{i j}^{(m, l)}=x_{i j}^{(l)}, i \in R_{l}, j \in R_{l} \cup\{l\}, l \in \mathcal{L} \\
& \sum_{k: i \in N_{l}(k)} x_{k i}^{(m, l)}=\sum_{j \in N_{l}(i)} x_{i j}^{(m, l)}, \\
& m \neq i ; m, i, k \in R_{l} ; j \in R_{l} \cup\{l\} ; l \in \mathcal{L} \\
& z_{l}\left(\sum_{j \in N_{l}(i)} x_{i j}^{(l)}-\sum_{k: i \in N_{l}(k)} x_{k i}^{(l)}\right)=w_{i}^{(l)} \text {, } \\
& i, k \in R_{l} ; j \in R_{l} \cup\{l\} ; l \in \mathcal{L} \\
& \left\{\sum _ { l = 1 } ^ { | \mathcal { L } | } z _ { l } \left(\sum_{k: i \in N_{l}(k)} \gamma \cdot x_{k i}^{(l)}+\right.\right. \\
& \left.\left.\sum_{j \in N_{l}(i)} C_{i j}^{(l)} \cdot x_{i j}^{(l)}\right)\right\} \cdot T \leq E_{i} \\
& i, k \in \mathcal{N} ; j \in \hat{\mathcal{N}} ; l \in \mathcal{L} \\
& \sum_{l: i \in R_{l}} w_{i}^{(l)}=D \cdot d_{i} \\
& x_{i j}^{(m, l)} \geq 0, \\
& i, m \in R_{l} ; j \in R_{l} \cup\{l\} ; l \in \mathcal{L}( \\
& w_{i}^{(l)} \geq 0, \\
& i \in R_{l}, l \in \mathcal{L} \\
& z_{l} \geq 0 \text {, } \\
& l \in \mathcal{L} \\
& T \geq 0 \text {. }
\end{aligned}
$$$$
i \in \mathcal{N}(25)
$$

In the sub-flow-based model, each node requires only $D \cdot d_{i}$ amount of buffer because there is no need to store other nodes' sub-flows.

\section{B. Queue-Based Model}

In the queue-based model, the sensor node can buffer the commodities or sub-flows of all nodes. Let $q_{i}^{(l)}$ be the queue length at node $i$ just before the sink moves from location $l$ to $l+1$. Assume that each node $i$ has $D \cdot d_{i}$ amount of data at the beginning of a cycle, which is denoted by $q_{i}^{(0)}$. When the sink finishes a cycle of visit, the queue at node $i$ must be cleared. Thus we have $q_{i}^{(|\mathcal{L}|)}=0$. In this model, the flow conservation constraint has to do with the queue length, expressed as follows.

$$
\begin{array}{r}
z_{l} \sum_{k: i \in N_{l}(k)} x_{i j}^{(l)}+q_{i}^{(l-1)}-z_{l} \sum_{j \in N_{l}(i)} x_{i j}^{(l)}=q_{i}^{(l)}, \\
i, k \in \mathcal{N} ; j \in \hat{\mathcal{N}} ; l \in \mathcal{L} .
\end{array}
$$

The energy constraints can be expressed in the same way as in the MSM or sub-flow based MSM. From the above discussion, we have the following optimization problem for maximizing the lifetime.

\section{Problem : Queue-Based DT-MSM}

$\max T$

$$
\begin{aligned}
& \text { s. t. } z_{l} \sum_{k: i \in N_{l}(k)} x_{k i}^{(l)}+q_{i}^{(l-1)}-z_{l} \sum_{j \in N_{l}(i)} x_{i j}^{(l)}=q_{i}^{(l)} \text {, } \\
& i, k \in \mathcal{N} ; j \in \hat{\mathcal{N}} ; l \in \mathcal{L} \\
& \left\{\sum _ { l = 1 } ^ { | \mathcal { L } | } z _ { l } \left(\sum_{k: i \in N_{l}(k)} \gamma \cdot x_{k i}^{(l)}+\right.\right. \\
& \left.\left.+\sum_{j \in N_{l}(i)} C_{i j}^{(l)} \cdot x_{i j}^{(l)}\right)\right\} \cdot T \leq E_{i} \\
& i, k \in \mathcal{N} ; j \in \hat{\mathcal{N}} ; l \in \mathcal{L} \\
& i \in \mathcal{N}, j \in \hat{\mathcal{N}}, l \in \mathcal{L} \\
& x_{i j}^{(l)} \geq 0, \\
& l \in \mathcal{L}, i \in \mathcal{N} \\
& q_{i}^{(l)} \geq 0 \text {, } \\
& i \in \mathcal{N} \\
& q_{i}^{(0)}=D \cdot d_{i}, \\
& i \in \mathcal{N} \\
& q_{i}^{(|\mathcal{L}|)}=0, \\
& l \in \mathcal{L} \\
& z_{l} \geq 0 \text {, } \\
& T \geq 0 \text {. }
\end{aligned}
$$

The problem shown above can be converted into an LP problem by substituting $y_{i j}^{(l)}$ for $z_{l} \cdot x_{i j}^{(l)}$ and introducing the new variable $u=1 / T$. This linearization method can also be applied to the MSM.

\section{Properties of Delay-Tolerant Mobile Sink Model}

To facilitate later expositions, we define the inclusion relationship between different optimization problems with the same decision variables $x$ and the same objective function $f(x)$ but different constraint sets. Without loss of generality, we consider the minimization problem.

Definition 1 (Inclusion). Consider two minimization problems $P_{1}$ and $P_{2}$ that have the same decision variables $x$ and 
the same objective function $f$ of variables $x$, but different constraints set $F_{1}$ and $F_{2}$, respectively. If $F_{1} \subseteq F_{2}$, we say that $P_{1}$ is said to be included in $P_{2}$ and denote $P_{1} \subseteq P_{2}$.

If $P_{1} \subseteq P_{2}$, we can always obtain better optimal solution from $P_{2}$ than $P_{1}$. In general, we say that inclusion holds for a sequence of optimization problems $\left\{P_{i}\right\}$, where $i$ is from some linearly ordered index set, $I$, that is, $P_{a} \subseteq P_{b}$ whenever $a<b, a, b \in I$.

In both sub-flow-based model and queue-based model, the coverage of the sink location is a very important factor for the lifetime of the WSN. Consider the optimization problem formulated for the sub-flow-based model. Depending on the radius of coverage, we may obtain different instances of the optimization problem. This observation is also valid in the queue-based model. Thus, we can parameterize the optimization problems according to the radius of the coverage. Let $P(\mathcal{N}, \mathcal{L}, r)$ be the optimization problem when the radius of coverage of the sinks is $r$, the set of sensor nodes is $\mathcal{N}$, and the set of sink locations is $\mathcal{L}$. The value $r$ must be large enough so that all sensor nodes can be covered by at least one sink location and we denote this minimum radius of coverage for connectivity by $r_{0}$. Under the same configuration with $\mathcal{N}$ and $\mathcal{L}$, different $r$ values only affects $N_{l}(i)$ and $C_{i j}^{(l)}$. We will use the notation $N_{l}(i, r)$ and $C_{i j}^{(l)}(r)$ if it is necessary to specify the radius of coverage. In the next theorem, we prove that the bigger the radius of coverage,the longer the optimal lifetime is. For the proof of this theorem, we need the following lemma.

Lemma 2. The sequence $\{P(\mathcal{N}, \mathcal{L}, r)\}, r \in \mathbb{R}$, satisfies inclusion. That is, $P\left(\mathcal{N}, \mathcal{L}, r_{1}\right) \subseteq P\left(\mathcal{N}, \mathcal{L}, r_{2}\right)$ for any pair of $r_{1}, r_{2}$ such that $r_{1}<r_{2}$.

Proof: Let $G=\{\mathcal{N} \cup \mathcal{L}, E\}$ be a graph where $E=$ $\{(i, j) \mid i \in \mathcal{N}, j \in \mathcal{N} \cup \mathcal{L}, d(i, j) \leq c\}$, where $c$ is the transmission range of the sensor nodes. The neighbor set of node $i$ is defined as $N(i)=\{j \in \mathcal{N} \cup \mathcal{L} \mid(i, j) \in E\}$. Let $G(r)$ be the graph for the DT-MSM model with the radius $r$ and $G(r)=\left\{\mathcal{N} \cup \mathcal{L}, \cup_{l=1}^{|\mathcal{L}|} E^{(l)}(r)\right\}$, where $E^{(l)}(r)=\{(i, j) \mid i \in$ $\left.R_{l}, j \in R_{l} \cup\{l\}, d(i, j) \leq c\right\}$, and $R_{l}=\{i \in \mathcal{N} \mid d(i, l) \leq r\}$. The neighbor set of node $i$ when the sink is at $l, N_{l}(i)$ is $\left\{j \in R_{l} \cup\{l\} \mid(i, j) \in E^{(l)}(r)\right\}$.

Consider the two optimization problems $P\left(\mathcal{N}, \mathcal{L}, r_{1}\right)$ and $P\left(\mathcal{N}, \mathcal{L}, r_{2}\right)$ with $r_{1}<r_{2}$. It is obvious that $N_{l}\left(i, r_{1}\right) \in$ $N_{l}\left(i, r_{2}\right)$ for all $i \in \mathcal{N}, l \in \mathcal{L}$ by the definition of neighbor set $N_{l}(i, r)$. Therefore, we can split the larger set $N_{l}\left(i, r_{2}\right)$ into two sets $A$ and $\bar{A}$, where $A=N_{l}\left(i, r_{1}\right)$, and $\bar{A}=$ $N_{l}\left(i, r_{2}\right)-A$. In a similar way, we can also split the incoming neighbor set for the node $i$ into $B=\{k \in \mathcal{N} \mid(k, i) \in$ $E^{(l)}\left(r_{1}\right)$ and $\left.(k, i) \in E^{(l)}\left(r_{2}\right)\right\}$ and $\bar{B}=\{k \in \mathcal{N} \mid(k, i) \in$ $\left.E^{(l)}\left(r_{2}\right)\right\}-B$. In other words, $\bar{A}$ and $\bar{B}$ are the extended outgoing and incoming neighbor sets for node $i$, respectively, as the radius of coverage increases from $r_{1}$ to $r_{2}$.

Suppose that $(\hat{x}, \hat{w}, \hat{z}, \hat{T})$ is a feasible solution to the problem $P\left(\mathcal{N}, \mathcal{L}, r_{1}\right)$. Now, consider equation (23) for the optimization problem $P\left(\mathcal{N}, \mathcal{L}, r_{2}\right)$.

$$
z_{l}\left(\sum_{j \in N_{l}\left(i, r_{2}\right)} x_{i j}^{(l)}-\sum_{k: i \in N_{l}\left(k, r_{2}\right)} x_{k i}^{(l)}\right)=w_{i}^{(l)} .
$$

We have the following by separating the neighbor sets into $A, \bar{A}, B$, and $\bar{B}$.

$$
z_{l}\left(\sum_{j \in A} x_{i j}^{(l)}+\sum_{j \in \bar{A}} x_{i j}^{(l)}-\sum_{k \in B} x_{k i}^{(l)}-\sum_{k \in \bar{B}} x_{k i}^{(l)}\right)=w_{i}^{(l)},
$$

for $i, k \in \mathcal{N}, j \in \mathcal{N} \cup\{l\}, l \in \mathcal{L}$. We set the variable $x_{i j}^{(l)}=\hat{x}_{i j}^{(l)}$ when $j \in A$, and $x_{i j}^{(l)}=0$ when $j \in \bar{A}$. In addition, we set $x_{k i}^{(l)}=\hat{x}_{k i}^{(l)}$ when $k \in B$ and $x_{k i}^{(l)}=0$ when $k \in \bar{B}$. For the variables $w$ and $z$, let $w^{(l)}(i)=\hat{w}^{(l)}(i)$ and $z_{l}=\hat{z}_{l}$. Then, equation (41) is equivalent to the constraint (23) of the problem $P\left(\mathcal{N}, \mathcal{L}, r_{1}\right)$. Therefore, $(\hat{x}, \hat{w}, \hat{z}, \hat{T})$ satisfies the constraint (23) for the problem $P\left(\mathcal{N}, \mathcal{L}, r_{2}\right)$. For the energy constraint (24), we can also apply the similar procedures. Hence, we can conclude that any feasible solution of the problem $P\left(\mathcal{N}, \mathcal{L}, r_{1}\right)$ is also a feasible solution for the problem $P\left(\mathcal{N}, \mathcal{L}, r_{2}\right)$. Hence, $P\left(\mathcal{N}, \mathcal{L}, r_{1}\right) \subseteq P\left(\mathcal{N}, \mathcal{L}, r_{2}\right)$

As a consequence, we have the following theorem.

Theorem 3. Consider the set of maximization problem $\{P(\mathcal{N}, \mathcal{L}, r)\}, r \in \mathbb{R}$. If $r_{1}<r_{2}$, then optimal objective value for the problem $P\left(\mathcal{N}, \mathcal{L}, r_{2}\right)$ is greater than that for the problem $P\left(\mathcal{N}, \mathcal{L}, r_{1}\right)$.

In general, the queue-based model is less constraining than the sub-flow-based model. Naturally, this results in lifetime gains in the former model. The following theorem formalizes the fact that the queue-based model always outperforms the sub-flow-based model.

Theorem 4. Let $\hat{T}$ be the optimal objective value to problem (20), and $T^{*}$ be the optimal objective value to problem (31) with the same configuration $(\mathcal{N}, \mathcal{L})$ and the same radius of coverage $r$. Then $\hat{T} \leq T^{*}$.

Proof: Let $\left(\hat{x}_{i j}^{(m, l)}, \hat{w}_{i}^{(l)}, \hat{z}_{k}, \hat{T}\right)$ be the optimal solution to problem (20). We will prove this theorem by constructing a feasible solution to problem (31) with $\left(\hat{x}_{i j}^{(m, l)}, \hat{w}_{i}^{(l)}, \hat{Z}_{k}, \hat{T}\right)$, and showing that under this feasible solution, the objective value of problem (31) is the same. By $(21), x_{i j}^{(l)}=$ $\sum_{m \in R_{l}} \hat{x}_{i j}^{(m, l)}$. In addition, let set $q_{i}^{(l)}=q_{i}^{(l-1)}-w_{i}^{(l)}$ for all $i \in \mathcal{N}$. Since $q_{i}^{(0)}=D \cdot d_{i}$ by (36), we have the following sequence of assignments for the $q(\cdot)_{i}$.

$$
\begin{aligned}
q_{i}^{(1)} & =q_{i}^{(0)}-w_{i}^{(1)}=D \cdot d_{i}-w_{i}^{(1)} \\
q_{i}^{(2)} & =q_{i}^{(1)}-w_{i}^{(2)} \\
\vdots & \ddots \\
q_{i}^{(|\mathcal{L}|)}= & q_{i}^{(|\mathcal{L}|-1)}-w_{i}^{(|\mathcal{L}|)} .
\end{aligned}
$$

If we sum up above assignment for all $l \in \mathcal{L}$, we have $q_{i}^{(|\mathcal{L}|)}=D \cdot d_{i}-\sum_{l}^{|\mathcal{L}|} w_{i}^{(l)}=D \cdot d_{i}-D \cdot d_{i}=0$ by $(25)$ 
and it exactly coincides with (37). Since the configuration and radius of coverage $r$ for problem (20) are the same as problem (31), $N_{l}(i), i \in \mathcal{N}, l \in \mathcal{L}$ for (20) does not change in (31). If we put $w_{i}^{(l)}=q_{i}^{(l-1)}-q_{i}^{(l)}$ into (23), we have exactly the same constraint as (32). Energy constraint (24) is equivalent to the (33). From the above arguments, we conclude that for the optimal solution to problem (20), there exists a corresponding feasible solution to problem (31) with the same objective value. Hence, $T^{*} \geq \hat{T}$.

In the following theorem, we show that the maximum lifetime of the system is the same for all values of $D$. Here, the maximum lifetime of the system is expressed as the product of the $D$ and the corresponding optimal objective value $T^{*}(D)$.

Theorem 5. Define $P(D)$ as the lifetime optimization problem parameterized by the value $D$, for some fixed network configuration. Let $T^{*}(D)$ and $T^{*}\left(D^{\prime}\right)$ be the optimal objective values for the problem $P(D)$ and $P\left(D^{\prime}\right)$, respectively. Then, $T^{*}(D) \cdot D=T^{*}\left(D^{\prime}\right) \cdot D^{\prime}$.

Proof: Consider the queue-based model. Let $\left(x^{*}(D), q^{*}(D), z^{*}(D), T^{*}(D)\right)$ be the optimal solution to the problem $P(D)$, and let $\left(x^{*}\left(D^{\prime}\right), q^{*}\left(D^{\prime}\right), z^{*}\left(D^{\prime}\right), T^{*}\left(D^{\prime}\right)\right)$ be the optimal solution to the problem $P\left(D^{\prime}\right)$.

First, suppose $T^{*}(D) \cdot D<T^{*}\left(D^{\prime}\right) \cdot D^{\prime}$. We will show this contradicts the definition of the optimal value, $T^{*}(D)$ by constructing a feasible solution whose objective value is greater than $T^{*}(D)$ for the problem $P(D)$.

Let $x=\left(\frac{D}{D^{\prime}}\right) x^{*}\left(D^{\prime}\right), q=\left(\frac{D}{D^{\prime}}\right) q^{*}\left(D^{\prime}\right), z=z^{*}\left(D^{\prime}\right)$, $T=\left(\frac{D^{\prime}}{D}\right) T^{*}\left(D^{\prime}\right)$. We want to show that $(x, q, z, T)$ satisfies the constraints (32)-(39). Since it is obvious that the solution $(x, q, z, T)$ satisfies the constraints (34), (35), (37), (38), and (39), we focus here on constraints (32), (33), and (36) only. Since the optimal solution $\left(x^{*}\left(D^{\prime}\right), q^{*}\left(D^{\prime}\right), z^{*}\left(D^{\prime}\right), T^{*}\left(D^{\prime}\right)\right)$ is feasible to the problem $P\left(D^{\prime}\right)$, it must satisfy constraint (32). Next, let us plug $\left(\frac{D^{\prime}}{D}\right) x, z$, and $\left(\frac{D^{\prime}}{D}\right) q$ into constraint (32) in the places for $x^{*}\left(D^{\prime}\right), z^{*}\left(D^{\prime}\right)$, and $q^{*}\left(D^{\prime}\right)$, respectively. Then, we have

$$
z_{l} \sum_{k: i \in N_{l}(k)} x_{k i}^{(l)}+q_{i}^{(l-1)}-z_{l} \sum_{j \in N_{l}(i)} x_{i j}^{(l)}=q_{i}^{(l)} .
$$

If we put $\left(\frac{D^{\prime}}{D}\right) x, z$, and $\left(\frac{D}{D^{\prime}}\right) T$ in the places for $x^{*}\left(D^{\prime}\right)$, $z^{*}\left(D^{\prime}\right)$ and $T^{*}\left(D^{\prime}\right)$ on the left hand side of constraint (33), we have

$$
\begin{array}{r}
\left\{\sum _ { l = 1 } ^ { | \mathcal { L } | } z _ { l } \left(\sum_{k: i \in N_{l}(k)} \gamma \cdot x_{k i}^{(l)}\left(\frac{D^{\prime}}{D}\right)+\right.\right. \\
\left.\left.\sum_{j \in N_{l}(i)} C_{i j}^{(l)} \cdot x_{i j}^{(l)}\left(\frac{D^{\prime}}{D}\right)\right)\right\} \cdot T\left(\frac{D}{D^{\prime}}\right)
\end{array}
$$

After canceling $D$ and $D^{\prime}$, it is easy to see that the new solution $(x, q, z, T)$ satisfies the energy constraint of the problem $P(D)$.

From the constraint (36) for the problem $P\left(D^{\prime}\right)$, we have $q_{i}^{*(0)}\left(D^{\prime}\right)=D^{\prime} d_{i}$. Since $q=\left(\frac{D}{D^{\prime}}\right) q^{*}\left(D^{\prime}\right), q_{i}^{*(0)}\left(D^{\prime}\right)=$
TABLE I

EXPERIMENTAL PARAMETERS AND THEIR VALUES

\begin{tabular}{|l|l|}
\hline the number of sensor nodes & $\{100,200\}$ \\
\hline the number of possible sink locations & $\{5,6,7,8,9,10,15,20,30,40\}$ \\
\hline path loss exponent $(e)$ & $\{2.0,3.0\}$ \\
\hline transmission range & $\{5,6,7,8,9,10,15,20,30,40,50\}$ \\
\hline$\alpha$ & $10 \mathrm{pJ} / \mathrm{bit} / \mathrm{m}^{2}$ \\
\hline$\beta$ & $0.0013 \mathrm{pJ} / \mathrm{bit} / \mathrm{m}^{4}$ \\
\hline Initial Energy $\left(E_{i}\right)$ & $500 \mathrm{~J}$ \\
\hline Data generation rate $\left(d_{i}\right)$ & $500 \mathrm{bps}$ \\
\hline
\end{tabular}

$q\left(\frac{D^{\prime}}{D}\right)=D^{\prime} d_{i}$. Therefore we have

$$
q=D d_{i} .
$$

From above argument, we have shown that new solution $(x, q, z, T)$ is feasible to the problem $P(D)$. According to the assumption, we know $T^{*}\left(D^{\prime}\right)>\left(\frac{D}{D^{\prime}}\right) T^{*}(D)$. Therefore, we have

$$
T=\left(\frac{D^{\prime}}{D}\right) T^{*}\left(D^{\prime}\right)>\left(\frac{D^{\prime}}{D}\right)\left(\frac{D}{D^{\prime}}\right) T^{*}(D)=T^{*}(D)
$$

However, this is contradictory to the fact that $T^{*}(D)$ is the optimal value for the problem $P(D)$. Thus, it must be that $T^{*}(D) D \geq T^{*}\left(D^{\prime}\right) D^{\prime}$.

Similarly, we can also prove that $T^{*}(D) D \leq T^{*}\left(D^{\prime}\right) D^{\prime}$. Hence, $T^{*}(D) D$ must equal to $T^{*}\left(D^{\prime}\right) D^{\prime}$

\section{EXPERIMENTAL RESULTS}

In this section, we will present the results from numerical experiments. In particular we have compared the network lifetimes of the following models.

- Static Sink Model (SSM): The stationary sink is located at $O$. We take the performance of this model as the reference for comparison.

- Mobile Sink Model (MSM): The sink can move to several locations to collect data. When the sink is at each location, all sensors participate in the communication, sending and relaying traffic to the sink.

- Delay-Tolerant Mobile Sink Model (DT-MSM): When the mobile sink is at a stop, a subset of the sensor nodes can participate in the communication. We use queuebased variant of this model to evaluate the performance.

We have experimented with different parameters extensively, such as the number of nodes, the number of possible sink locations and the parameters for the energy consumption model. Only a small subset of the results are reported here due to space limitation. In Table I, we provide the system parameters and their values for the reported experiments in this paper. We adopt the data for the last four parameters from [20]. In all experiments, we use GLPK for solving the linear programming problem.

First, we would like to mention the impact of the radius of coverage of the sink on the performance of the DT-MSM. For this experiment, pointf for 100 nodes and 20 mobile-sink locations are randomly generated $(|\mathcal{N}|=100,|\mathcal{L}|=20)$ in a circular area with radius 25 . We use a simple algorithm 


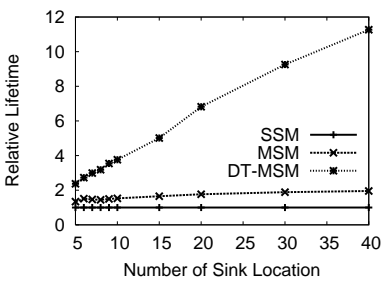

(a) 100-node network

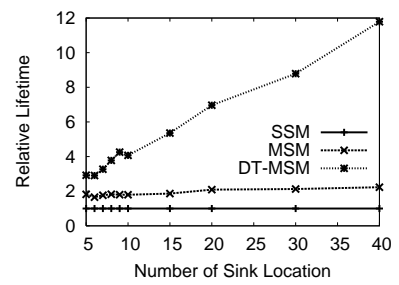

(b) 200-node network
Fig. 3. Lifetime against the number of sink locations; maximum coverage; $e=2.0$

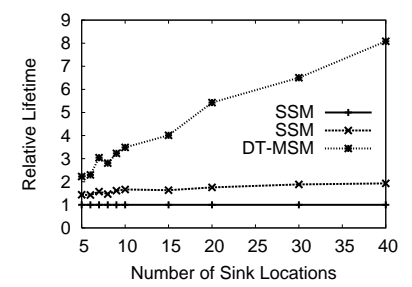

(a) 100-node network

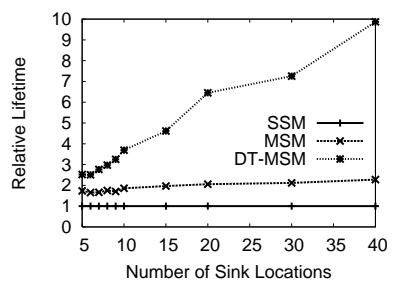

(b) 200-node network
Fig. 4. Lifetime against the number of sink locations; minimum coverage; $e=3.0$

to find the minimum radius of coverage (denoted by $r_{0}$ ): At each sink location, we increase the radius of coverage from 0 simultaneously until the union of all coverages contains all sensor nodes. At that point, we have reached the minimum radius of coverage required to cover all nodes. After that, we increase the radius of coverage in 0.1 increments. The result of the experiment is plotted in Figure 2. Note that, in the figure, the lifetime is normalized to the optimal lifetime of the MSM. As shown in the figure, the lifetime of the DT-MSM increases as the radius of coverage increases, which is consistent with Theorem 3. The increase is the sharpest when the radius just exceeds the minimum radius required to cover all nodes. After that, further increase of the radius has a negligible effect. Recall that, when the mobile sink reaches one of the stops, say $l$, only those sensor nodes in the coverage of $l$ (i.e., $R_{l}$ ) can communicate. It is generally desirable for $R_{l}$ to have as few nodes as possible, since this reduces the communication and coordination complexity. The aforementioned behavior of lifetime increase is desirable.

Next, we compare the lifetimes of models under various numbers of the sink locations. The number of nodes is set to 100 or 200 , the path loss exponent $e$ is 2.0 , and the coverage is as large as possible. We ran the experiment 100 times for each configuration. The lifetimes of the MSM and DT-MSM are again normalized to the optimal lifetime of the SSM. As shown in Figure 3, the lifetime of the MSM is about $100 \% \sim$ $200 \%$ greater than that of the SSM. However, the DT-MSM is $200 \% \sim 1000 \%$ better than the SSM. Moreover, the curves all look linear; the performance gap can grow even larger with more sink locations.

Interestingly, the lifetime of the MSM increases very slowly with the number of sink locations. As explained in [9], in the optimal solution, only a few locations from the set of sink

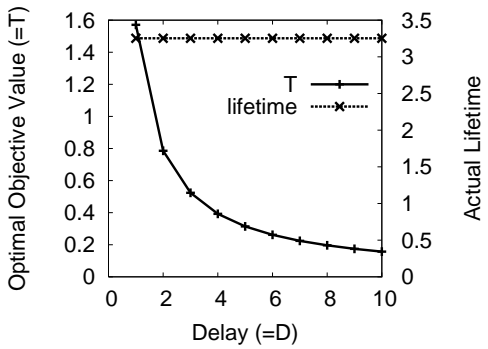

Fig. 5. Lifetime/optimal objective value $(T)$ versus the delay tolerance level D

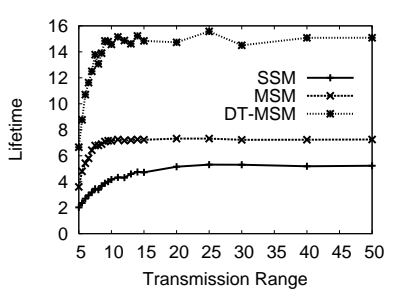

(a) Under minimum coverage

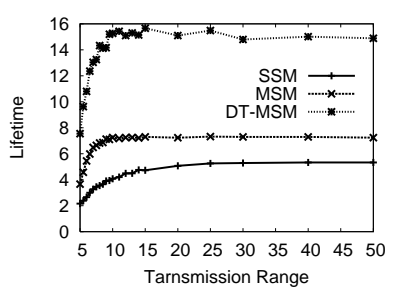

(b) Under maximum coverage
Fig. 6. Lifetimes versus transmission range: $|\mathcal{N}|=200,|\mathcal{L}|=20, e=2.0$

locations are chosen as the true stops for the sink. However, in DT-MSM, the rate of lifetime increase is substantial as $|\mathcal{L}|$ increases. This is because each node can have better and better sink location as $|\mathcal{L}|$ increases, and it is not forced to participate in the communication when the current coverage is not the most favorable for energy saving, even if the node may belong to that coverage. This is not possible in the MSM because no matter where the sink stops, every node must participate in the communication.

We conduct similar experiments with the same configuration but minimum coverage. The result is shown in Figure 4. Although the slope of lifetime increase of the DT-MSM is lowered when compared to the maximum coverage case, the increase pattern is similar. Although a larger set of sink locations increases the network lifetime, it can be undesirable if the sink travel time cannot be ignored. The longer traveling time may exceed the delay tolerance level $D$. Therefore, there is a tradeoff between the gain from more sink locations and the delay or other system costs.

We also conducted experiments with different values for the delay tolerance level $D$. With 100 nodes and 20 sink locations, we computed the optimal lifetime of the queue-based model as $D$ varies from 1 to 10 . In Figure 5, we plot the optimal objective values $T$ (number of cycles) and the actual optimal lifetime of the system. Note that the actual lifetime of the system is $T \times D$. As claimed by Theorem 5, the optimal lifetime is a constant regardless of the values of $D$.

In Figure 6, we show the lifetimes of the three models under various values for the transmission range. The transmission range determines whether a link exists between a pair of nodes. For the minimum radius of coverage of the sink (See Figure 6(a)), the effective transmission range limited by the dimension of the coverage of the sink. In other words, a node cannot 
use a link to another node if they are not in the common coverage. Both the MSM and the DT-MSM exhibit a sharp lifetime increase when the transmission range is small but increasing. However, the transmission range becomes large, the lifetime increase comes to a stop for all 3 models. The result implies that long-distance wireless links are not beneficial for improving the system lifetime.

\section{CONCLUSION}

In this paper, we proposed a new framework for using the mobile sink to improve the network lifetime. It is expected to be useful in applications that can tolerate a certain amount of delay in data delivery. We presented the mathematical formulation for optimizing the network lifetime under the proposed framework. We identified several properties that our model possesses. In particular, we showed the delay tolerance level does not affect the optimal system lifetime. To validate the proposed framework, we conducted extensive experiments and found that the proposed framework is superior to the models published previously. The lifetime gain of the proposed model is significant when compared to other models. Furthermore, as the number of sink locations increases, the optimal network lifetime increases substantially. The results of the paper can be both applied to practical situations and can be used as benchmarks for studying energy-efficient network design.

\section{REFERENCES}

[1] Z. M. Wang, S. Basagni, E. Melachrinoudis, and C. Petrioli, "Exploiting sink mobility for maximizing sensor network lifetime," in 38th Hawail International Conference on System Science, 2005.

[2] M. Gatzianas and L. Georgiadis, "A distributed algorithm for maximum lifetime routing in sensor networks with mobile sink," IEEE Transactions on Wireless Communications, vol. 7, no. 3, pp. 984-994, March 2008.

[3] J. Luo and J.-P. Hubaux, "Joint mobility and routing for lifetime elongation in wireless sensor networks," in INFOCOM 05, 2005.

[4] R. C. Shah, S. Roy, S. Jain, and W. Brunette, "Data mules: Modeling a three-tier architecture for sparse sensor networks," in the First IEEE International Workshop on Sensor Network Protocols and Applications, SNPA 2003, Anchorage, AK, May 2003, pp. 30-41.

[5] S. Basagni, A. Carosi, E. Melachrinoudis, C. Petrioli, and Z. M. Wang, "A new milp formulation and distributed protocols for wireless sensor networks lifetime maximization," in IEEE International Conference on Communications 2006, June 2006, pp. 3517-3524.

[6] W. Wang, V. Srinivasan, and K.-C. Chua, "Using mobile relays to prolong the lifetime of wireless sensor networks," in MobiCom '05, 2005, pp. 270-283.

[7] I. Papadimitriou and L. Georgiadis, "Maximum lifetime routing to mobile sink in wireless sensor networks," in The 13th IEEE SoftCom, 2005, 2005.

[8] S. Gandham, M. Dawande, R. Prakash, and S. Venkatesan, "Energy efficient schemes for wireless sensor networks with multiple mobile base stations," in Global Telecommunications Conference, 2003. GLOBECOM '03. IEEE, December 2003.

[9] Y. Shi and Y. T. Hou, "Theoretical results on base station movement problem for sensor network," in IEEE INFOCOM '08, 2008.

[10] L. Popa, A. Rostamizadeh, R. M. Karp, and C. Papadimitriou, "Balancing traffic load in wireless networks with curveball routing," in MobiHoc '07, Sept 2007, pp. 170-179.

[11] J. Li and P. Mohapatra, "An analytical model for the energy hole problem in many-to-one sensor networks," in Proc. of Vehicular Technology Conference, January 2005, pp. 2721- 2725.

[12] — - "Analytical modeling and mitigation techniques for the energy hole problem in sensor networks," Pervasive and Mobile Computing, vol. 3, no. 8, pp. 233-254, 2007.
[13] S. K. D. Xiaobing Wu, Guihai Chen, "Avoiding energy holes in wireless sensor networks with nonuniform node distribution," To be appeared on IEEE Transactions on Parallel and Distributed Systems, 2007.

[14] W. R. Heinzelman, A. Chadrakasan, and H. Balakrishnan, "Energyefficient communication protocol for wireless microsensor networks," in Proc. of the 33rd Hawaii International Conference on System Sciences, January 2000.

[15] T. S. Rappaport, Wireless communications: principles and practice. Prentice Hall, 1996.

[16] J. Chang and L. Tassiulas, "Routing for maximum system lifetime in wireless ad hoc networks," in 37th Annual Allertion Conf. Communication, Control, and Computing, Monticello, IL, September 1999.

[17] — "Maximum lifetime routing in wireless sensor networks," IEEE/ACM Transactions of Networking, vol. 12, pp. 609-619, August 2004.

[18] R. K. Ahuja, T. L. Magnanti, and J. B. Orlin, Network Flows: Theory, Algorithms, and Applications. Prentice Hall, 1993.

[19] N. Garg and J. Konemann, "Faster and simpler algorithms for multicommodity flow and other fractional packing problems," in Proc. 39th Annual Symposium on Foundations of Computer Science, 1998, pp. 300309.

[20] W. B. Heinzelman, "Application specific protocol architectures for wireless networks," Ph.D. dissertation, MIT, 2000 